\title{
Inverse Operation of Four-dimensional Vector Matrix
}

\author{
H J Bao \\ School of Communication Engineering, Jilin University, Changchun, China \\ Email: baohj09@mails.jlu.edu.cn \\ A J Sang and H X Chen \\ School of Communication Engineering, Jilin University, Changchun, China \\ Email:sangaj@jlu.edu.cn \\ Email: chenhx@jlu.edu.cn
}

\begin{abstract}
This is a new series of study to define and prove multidimensional vector matrix mathematics, which includes four-dimensional vector matrix determinant, fourdimensional vector matrix inverse and related properties. There are innovative concepts of multi-dimensional vector matrix mathematics created by authors with numerous applications in engineering, math, video conferencing, 3D TV, and other fields.
\end{abstract}

Index Terms - multidimensional vector matrix, fourdimensional vector matrix determinant, four-dimensional vector matrix inverse

\section{INTRODUCTION}

This paper brings a new branch of mathematics called multidimensional vector matrix mathematics and its new subsets, four-dimensional vector matrix determinant and four-dimensional vector matrix inverse. The traditional matrix mathematics [1] that engineering, science, and math students are usually introduced to in college handles matrices of one or two dimensions. Ashu M. G. Solo [2] also defined some multidimensional matrix algebra operations. Multidimensional matrix mathematics extends the classical matrix mathematics to any figure of dimensions.

Matrix inversions are very significant means in many fields of combinatory and special functions principle. When dealing with combinatorial sums, application of matrix inversion may help to simplify problems, or propose new identities. At this point it seems suitable to set forth a little on the history of matrix inversions and inverse relations, specifically, since $\mathrm{H}$. W. Gould's name is inevitably tied with it [7]. In fact, Riordan provided lists of known matrix inversions and devoted two chapters of his book to inverse relations and their

Manuscript received January 15, 2011; revised February 24, 2011; accepted March 15, 2011.

Project number: The National Science Foundation of China under Grant 60911130128 and 60832002.

Corresponding author: Hexin Chen, School of Communication Engineering, Jilin University, Changchun 130022, China;

Email: chenhx@jlu.edu.cn application in his book [8] in the 1960s. As time passes, people brought to light an increasing number of such explicit matrix inversions. Gould spared no effort to study an outstanding part of these inverse relations in a series of papers [9], [10], [11], [12]. This study culminated in the important discovery, jointly with Hsu, of a very general matrix inversion [13], which possessed a great deal of inverse relations and properties of, what is currently called, Gould-type and Abel-type as special cases on matrix inversion [7].

The problem detected a q-analogue of their equation was immediately settled henceforth by Carlitz [20]. The importance of Carlitz' matrix inversion firstly showed up when Andrews [14] dug up that the Bailey transform [15], [16] is equivalent to a certain matrix inversion that is just a very unusual condition of Carlitz'. The Bailey transform is one of the corner stones in the development of the theory of hyper geometric series, corresponding to the inversion of two infinite lower-triangular matrices. However, Carlitz did not propose any applications of related definitions even earlier. A few years later, Gasper and Rahman proved a bibasic extension of that matrix inversion [17], [18], which unifies the matrix inversions of Gessel and Stanton, and Bressoud. Gessel and Stanton [6] used it to derive a great number of basic hyper geometric summations and transformations, and identities of Rogers-Ramanujan type. The end of this line of development came with the attempt of the first author to combine all these recent matrix inversions into one formula. Indeed, in 1989, he discovered a matrix inversion, published in [19], which subsumes most of Riordan's inverse relations and all the other aforementioned matrix inversions, as it contains them all as special cases [7]. Based on these theories and papers, multidimensional vector matrix extends traditional matrix math to any figure of dimensions. Therefore, the traditional matrix mathematics is a subset of multidimensional vector matrix mathematics.

This paper mainly brings forward the definition of four-dimensional vector matrix determinant and the fourdimensional vector matrix inverse. We adopt the form that is different from the definition of two-dimensional matrix. But the properties of two-dimensional matrix 
determinant and inverse can be extended to the fourdimensional vector matrix. The extension of classical matrix mathematics to any figure of dimensions has various applications in many branches of engineering, math, image compression, coding and other fields. We should promote the other applications of multidimensional vector matrix math that could not be done without his multidimensional vector matrix mathematics.

Our group has proposed the definition of multidimensional vector matrix, multiplication of multidimensional vector matrices, multidimensional Walsh orthogonal transform and traditional discrete cosine transform [3]. The multidimensional vector matrix model will reduce the time redundancy, space redundancy and color redundancy. Their application in color image compression and coding is more and more common and widespread. For one thing, it conquers the restriction of traditional two-dimensional matrix multiplication. For another thing, it carries on high efficiency of traditional matrix transform in the aspect of removing redundancy of color space. By means of multi-dimensional vector matrix model, color image data can be expressed and processed in a unified mathematical model, and better compression results are received.

In Section 2, a multi-dimensional vector matrix model will be introduced, and the related properties will be discussed. In Section 3, we will propose the definitions of four-dimensional vector matrix determinant and inverse. Verification the truth of formula with regard to the four-dimensional vector matrix determinant and inverse will be also given in the same Section. In Section 4 , the related properties of four-dimensional vector matrix determinant and inverse will be introduced. Section 5 concludes this paper.

\section{PROPOSED THEORY}

Based on the multidimensional vector matrix definition proposed by our group, we will further study four-dimensional vector matrix adjoin matrix, determinant, inverse matrix and related properties. Therefore, nothing more than the basic definition is presented.

\section{A. The Definition of Multi-dimensional Vector Matrix:}

An array of numbers $\left(a_{i_{1} i_{2}}\right)$ in two directions (one direction has $\mathrm{M}$ entries and the other direction has $\mathrm{N}$ entries) is called two-dimensional matrix, and the set of all such matrices is represented as $M_{M \times N}$. An array of numbers $\left(a_{i_{1} i_{2} \cdots i_{n}}\right)$ in $\mathrm{n}$ directions (each direction has $I_{i}$ entries, $1 \leq i \leq n$. $I_{i}$ can be called the order in this direction) is called multi-dimensional matrix, and the set of all such matrices is denoted as $M_{I_{1} \times I_{2} \times \cdots \times I_{n}}$ [4].

If the dimensions of multi-dimensional matrix $M_{K_{1} \times K_{2} \times \cdots \times K_{r}}$ are separated into two sets and the matrix is denoted as $M_{I_{1} \times I_{2^{\times \cdots}}} \rightarrow \underset{I_{m} \times J_{1 \times J}}{\rightarrow} \underset{2^{\times \cdots \times}}{ }$, where $m+n=r$.
$M_{I_{1} \times I 2^{\times} \cdots I_{m} \times J_{1} \times J_{2} \times \cdots \times J_{n}} \underset{ }{\rightarrow}$ can be denoted as $M_{I J}$, where I and $\mathrm{J}$ are for the vectors, $I=\left(I_{1}, I_{2}, \ldots, I_{m}\right), J=\left(J_{1}, J_{2}, \ldots, J_{n}\right)$. $M_{I_{1} \times I_{2} \times \cdots I_{m} \times J_{1} \times J_{2} \times \cdots \times J_{n}} \underset{\sim}{\rightarrow}$ can be called multi-dimensional vector matrix separated according to the vector I, and J, multidimensional vector matrix in short [4].

A multi-dimensional matrix has various relevant multi-dimensional vector matrices, whereas a multidimensional vector matrix has unique relevant multidimensional matrix.

\section{B. Multi-dimensional Vector Identity Matrix:}

Let $A_{I J}$ be a multidimensional vector matrix, where $I=\left(I_{1}, I_{2}, \ldots, I_{m}\right), J=\left(J_{1}, J_{2}, \ldots, J_{n}\right)$. If vector $I=J$, then $A_{I J}$ is called multidimensional vector square matrix [5].

Let $\delta_{i j}=\left\{\begin{array}{ll}1 & i=j \\ 0 & i \neq j\end{array}, \quad\right.$ where $i=j$ represents vector $I=\left(I_{1}, I_{2}, \ldots, I_{m}\right), J=\left(J_{1}, J_{2}, \ldots, J_{n}\right), \quad$ if it has the same dimension, the meanings of $i=j$ is that $m=n$, and $i_{1}=j_{1}, i_{2}=j_{2}, \ldots . i_{m}=j_{n}$.

If $A_{I J}=\left(\delta_{i j}\right), A_{I J}$ is said to be multi-dimensional vector identity matrix, denoted as $E_{I I}$, or $E$ simply.

\section{Equality of Multi-dimensional Vector Matrices:}

$$
\text { If both } A_{I_{1} \times I_{2} \times \ldots \times I_{n}}=\left(a_{i 1} i_{2} \ldots i_{n}\right)_{I 1^{\times} I_{2} \times \ldots \times I_{n}} \quad \text { and }
$$

$B_{I 1^{\times} I_{2} \times \ldots \times I_{n}}=\left(b_{i 1 i 2 \ldots i_{n}}\right)_{I 1^{\times} I_{2} \times \ldots \times I_{n}}$ are multi-dimensional matrices of $I_{1} \times I \times \ldots \times I_{n}$ orders, and the corresponding entries of them are equal, that is, $a_{i_{1} i_{2} \ldots i_{n}}=b_{i_{1} i_{2} \ldots i_{n}} \quad\left(1 \leq_{i_{1}} \leq I_{1} ; 1 \leq_{i_{2}} \leq I_{2} ; \ldots ; 1 i_{i_{n}} \leq I_{n}\right)$, then $A_{I 1^{\times} I_{2} \times \ldots \times I_{n}}$ is said to be equal to $B_{I 1^{\times} I_{2} \times \ldots \times I_{n}}$, which is denoted as $\mathrm{A}=\mathrm{B}$ [4].

If we suppose $I_{1}=I_{2}=\ldots=I_{n}$, then multi-dimensional matrix $A_{I 1^{\times} I_{2} \times \ldots \times I_{n}}$ is called multidimensional square matrix.

A multi-dimensional matrix is called zero multidimensional matrix if all its entries are zeroes, and is denoted as zero.

If the corresponding multi-dimensional matrices of two multi-dimensional vector matrices are equal, then the others two multi-dimensional vector matrices are also equal.

\section{Addition of Multi-dimensional Vector Matrices:}

Let

$$
A_{I 1^{\times I} 2^{\times \ldots \times I_{n}}}=\left(a_{i 1 i 2 \ldots i_{n}}\right)_{I_{1} \times I_{2} \times \ldots \times I_{n}}
$$
and

$B_{I 1^{\times} I_{2} \times \ldots \times I_{n}}=\left(b_{i 1 i_{2} \ldots i_{n}}\right)_{I 1^{\times} I_{2^{\times}} \times \times I_{n}}$ be two multi-dimensional matrices of $I_{1} \times I_{2} \times \ldots \times I_{n}$ orders. A multi-dimensional matrix $C_{I 1^{\times} I \times \ldots \times I_{n}}$ of $I_{1} \times I^{2 \times \ldots \times I_{n}}$ orders is called the sum of $A_{I 1^{\times} I_{2} \times \ldots \times I_{n}}$ and $B_{I 1^{\times} I_{2} \times \ldots \times I_{n}}$.

$$
C_{I_{1} \times I_{2} \times \ldots \times I_{n}}=\left(c_{i 1 i_{2} \cdots i_{n}}\right)=\left(a_{i 1 i 2 \cdots i_{n}}+b_{i 1 i 2 \cdots i_{n}}\right)_{I_{1} \times I_{2} \times \ldots \times I_{n}},
$$


Which is denoted as,

$$
C_{I 1^{\times} I_{2 \times \ldots \times I_{n}}}=A_{I 1^{\times} I_{2} \times \ldots \times I_{n}}+B_{I 1^{\times I} 2^{\times \ldots \times I_{n}}} .
$$

The sum of two multi-dimensional matrices is their entry-by-entry sum. So we can find that the dimensions of these two multi-dimensional matrices must be the same, and the order number of each dimension must be the same [4].

Because the addition of multi-dimensional matrices can be formulated as an addition between their entries, that is the addition of numbers. So it is not difficult to verify the properties as follows.

For any multi-dimensional matrix $A_{I 1^{\times} \times I_{2} \times \ldots \times I_{n}}$,

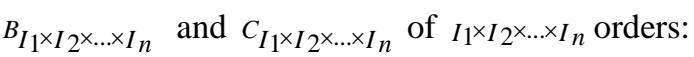

$$
\begin{aligned}
& \left(A_{I 1^{\times} I_{2} \times \ldots \times I n}+B_{I 1^{\times} I_{2} \times \ldots \times I_{n}}\right)+C_{I 1^{\times} I_{2} \times \ldots \times I_{n}} \\
& =A_{I 1^{\times} I_{2} \times \ldots \times I n}+\left(B_{I 1^{\times} I_{2} \times \ldots \times I n}+C_{I 1^{\times} I_{2} \times \ldots \times I_{n}}\right) \\
& \text { - } A_{I 1^{\times} I_{2} \times \ldots \times I_{n}}+B_{I 1^{\times} I_{2} \times \ldots \times I_{n}}=B_{I 1^{\times} I_{2} \times \ldots \times I_{n}}+A_{I 1^{\times} I_{2} \times \ldots \times I_{n}} \\
& \text { - } A_{I 1^{\times} I_{2} \times \ldots \times I_{n}}+0=A_{I 1^{\times I} 2^{\times \ldots \times I_{n}}}, \quad 0 \text { is zero multi- }
\end{aligned}
$$
dimensional matrix of $I_{1} \times I_{2^{\times \ldots \times}} \times I_{n}$ orders.

- Multi-dimensional matrix $\left(-a_{i 1 i 2 \ldots i n}\right)_{I 1^{\times} I_{2} \times \ldots \times I_{n}}$ is called negative multi-dimensional matrix of $A_{I 1^{\times} I_{2} \times \ldots \times I_{n}}=\left(a_{i 1 i_{2} \cdots i_{n}}\right)_{I 1^{\times} I_{2} \times \ldots \times I_{n}} \quad, \quad$ denoted by $-A_{I 1^{\times} \times I^{\times} \times \ldots \times I_{n}}$.

Obviously, $A_{I 1^{\times} I_{2} \times \ldots \times I_{n}}+\left(-A_{I 1^{\times} I_{2} \times \ldots \times I_{n}}\right)=0$.

Now that we define the concept of negative matrix of multi-dimensional matrix, we can conclude the subtraction:

$A_{I 1^{\times} I_{2} \times \ldots \times I n}-B_{I 1^{\times}} \operatorname{I2}^{\times \ldots \times I_{n}}=A_{I 1^{\times} I_{2} \times \ldots \times I n}+\left(-B_{I 1^{\times} I_{2} \times \ldots \times I n}\right)$.

Similarly,

$$
\begin{aligned}
& A_{I 1^{\times} I_{2} \times \ldots \times I_{n}}+B_{I 1^{\times I} 2^{\times \ldots \times}}=I_{n}=C_{I 1^{\times} I_{2} \times \ldots \times I_{n}} \\
\Leftrightarrow & B_{I 1^{\times} I_{2} \times \ldots \times I_{n}}=C_{I 1^{\times} I_{2} \times \ldots \times I_{n}}-A_{I 1^{\times} I_{2} \times \ldots \times I_{n}}
\end{aligned} .
$$

\section{E. Scalar Multiplication of Multi-dimensional Vector Matrices}

A multi-dimensional matrix $\left(m a_{i 1 i 2 \ldots i n}\right)_{I 1^{\times} I_{2 \times \ldots \times I_{n}}}$ is called the result of scalar multiplication of multidimensional matrix $A_{I 1^{\times} I_{2} \ldots \times I_{n}}=\left(a_{i 1 i_{2} \ldots i_{n}}\right)_{I_{1} \times I_{2} \times \ldots \times I_{n}}$ and real number $m$, denoted as $m A_{I 1^{\times} \times 2^{\times} \ldots \times I_{n}}$.

Scalar-multiplying a matrix is to multiply each entry of that matrix by $m$.

It is not difficult to verify the properties as follows:

$\cdot(m+n) A_{I 1^{\times} I_{2} \times \ldots \times I_{n}}=m A_{I 1^{\times} I_{2} \times \ldots \times I_{n}}+n A_{I 1^{\times} I_{2} \times \ldots \times I_{n}}$

- $m\left(A_{I 1^{\times} I_{2} \times \ldots \times I_{n}}+B_{I 1^{\times} I_{2} \times \ldots \times I_{n}}\right)=m A_{I 1^{\times} I_{2} \times \ldots \times I_{n}}+m B_{I 1^{\times} I_{2} \times \ldots \times I_{n}}$

$$
\text { - } m\left(n A_{I 1^{\times} I_{2} \times \ldots \times I_{n}}\right)=m n A_{I 1^{\times} I_{2} \times \ldots \times I_{n}}
$$

Where $m$ and $n$ are any numbers, and $A_{I 1^{\times} \times 2^{\times \ldots \times}}$ and $B_{I 1^{\times} \times 2^{\times} \times \times I_{n}}$ are any multi-dimensional matrices of $I_{1} \times I_{2} \times \ldots \times I_{n}$ orders.

For multi-dimensional matrix and multi-dimensional vector matrix, the results of equality, addition and scalar multiplication operation are the same. But for the operations followed, we must partition the dimensions of a multidimensional matrix into two parts, each of which is taken as a vector.

\section{F. Multiplication of Multi-dimensional Vector Matrices}

Let $A_{I J}$ and $B_{U V}$ be two multi-dimensional vector matrices, in which $I=\left(I_{1}, I_{2}, \ldots, I_{m}\right), J=\left(J_{1}, J_{2}, \ldots, J_{n}\right)$, $U=\left(U_{1}, U_{2}, \ldots, U_{S}\right), \quad V=\left(V_{1}, V_{2}, \ldots, V_{t}\right)$, If $J=U$, then $A_{I J}$ and $B_{U V}$ are multiplicative.

Let $A_{I L}$ be $I \times L$ matrix and $B_{L J}$ be $L \times J$ matrix. The result of multiplication of $A_{I L}$ and $B_{L J}$ is defined as a

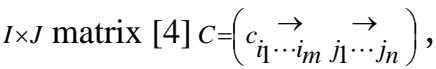

$$
c_{i_{1} \cdots i_{m} j_{1}, \cdots j_{n}}=\sum_{\mathbf{L}} a_{\mathrm{il}} b_{\mathbf{l j}}=\sum_{l_{1}}^{L_{1}} \cdots \sum_{l_{k}}^{L_{k}} a_{i_{1} \cdots i_{m} l_{1} \cdots l_{k}} \underset{l_{1} \cdots l_{k}, j_{1} \cdots j_{n}}{\longrightarrow}
$$

Which is denoted as $C_{I J}=A_{I L} B_{L J}$.

For simplicity, the signal $\sum_{l_{1}}^{L_{1}} \cdots \sum_{l_{k}}^{L_{k}}(\cdots)$ is rewritten as $\sum_{L}(\cdots)$ and the signal $a_{i_{1} i_{2} \cdots i_{m} l_{1} l_{2} \cdots l_{k}} \rightarrow$ is rewritten as $a_{i l}$. If no specified, this kind of form is default.

Several items are worth the whistle in the multiplication of matrices:

1. Matrices multiplication is not commutative. In matrix multiplication, multiplier and multiplicand are not commutative. The main reason is that $B_{U V} A_{I J}$ may not make sense when $A_{I J} B_{U V}$ makes sense. Moreover, even though both of them make sense, they may not be equal. But in some cases, $A_{I J} B_{U V}$ may be equal to $B_{U V} A_{I J}$. When $A_{I J} B_{U V}=B_{U V} A_{I J}, A_{I J}$ and $B_{U V}$ are said to be commutative. Obviously, commutative matrices must be square matrices with the same orders.

2. The cancellation law of multiplication does not hold. When $A_{I L} B_{L J}=A_{I L} C_{L U}$, it must not be deduced that $B_{L J}=C_{L U}$.

3. The multiplication of two non-zero matrices may be zero matrix.

\section{G. Multi-dimensional Vector Matrix Transpose}

The definition of multi-dimensional vector matrix transpose

$$
A_{I J}{ }^{T}=A_{J I}
$$

For any matrices $A_{I J}$ and $B_{I J}$, there are some properties as follows: 
- $\left(A_{I J}^{T}\right)^{T}=A I J$

- $\left(A_{I J}^{T}+B_{I J}^{T}\right)^{T}=A_{I J}{ }^{T}+B_{I J}{ }^{T}=A_{J I}+B_{J I}$

- $\left(m A_{I J}\right)^{T}=m A_{I J}{ }^{T}=m A_{J I}$

- $\left(A_{I J}^{T} B_{I J}^{T}\right)^{T}=B_{I J}^{T} A_{I J}^{T}=B_{J I} A_{J I}$

H. Kronecker Multiplication of Multi-dimensional Vector Matrices

Let

$$
A_{I 1^{\times} I_{2} \times \ldots \times I_{n}}=\left(a_{i 1 i_{2} \cdots i_{n}}\right)_{I 1^{\times} I_{2} \times \ldots \times I_{n}}
$$

and

$B_{J_{1} \times J_{2} \times \ldots \times J_{n}}=\left(b_{j_{1} j_{2} \ldots j_{n}}\right)_{J_{1} \times J_{2} \times \ldots \times J_{n}}$ be two multi-

dimensional matrices, whose dimensions are the same. The block matrix $A \otimes B=\left(a_{i_{1} i_{2} \cdots i_{n}}\right)_{I_{1} J_{1} \times I_{2} J_{2} \times \ldots \times I_{n} J_{n}}$ is called the Kronecker multiplication of $A_{I 1^{\times} \times 2_{2} \times \ldots \times I_{n}}$ and $B_{J_{1} \times J_{2} \times \ldots \times J_{n}}$. The symbol $\otimes$ denotes Kronecker multiplication of multi-dimensional matrices. It is obvious that the Kronecker multiplication is only relative to the sequence of data and not the mode where the dimensions are partitioned [4].

The nuclear matrix of $2 \mathrm{M}$-dimensional vector matrix orthogonal transformation,

$$
\begin{aligned}
& C_{I J}=\left(c_{u_{1} u 2 \cdots u_{M} v_{1} v_{2} \cdots v_{M}}\right) \\
& I=\left(N_{1}, N_{2}, \ldots, N_{M}\right), J=\left(N_{1}, N_{2}, \ldots, N_{M}\right) \\
& c_{u 1} u_{2} \cdots u_{M} v_{1} v_{2} \cdots v v_{M} \\
& \text {, }=\left(\frac{2^{M}}{N_{1} N_{2} \cdots N_{M}}\right)^{\frac{1}{2}} c\left(u_{1}\right) c\left(u_{2}\right) \ldots c\left(u_{M}\right) \\
& \times \cos \frac{\left(2 v 1^{+1}\right) u 1^{\pi}}{2 N_{1}} \cos \frac{\left(2 v_{2}+1\right) u 2^{\pi}}{2 N_{2}} \ldots \cos \frac{\left(2 v_{M}+1\right) u_{M} \pi}{2 N_{M}} \\
& c\left(u_{\mathrm{i}}\right)= \begin{cases}\frac{1}{\sqrt{2}} & u_{i}=0 \\
1 & u_{i}=\text { others }\end{cases} \\
& u_{i}=0,1, \ldots, N_{1}-1 \quad v_{i}=0,1, \ldots, N_{1}-1 \\
& u_{i}=0,1, \ldots, N_{i}-1 \quad v_{i}=0,1, \ldots, N_{i}-1, M, N_{i} \in N^{*}, i=1,2, \ldots, M
\end{aligned}
$$

Based on these theories of multidimensional vector matrix and definitions of two-dimensional matrix determinant, we will further define the four-dimensional vector matrix determinant and inverse.

\section{FOUR-DIMENSIONAL VECTOR MATRIX DETERMINANT AND INVERSE}

The multidimensional vector matrix determinant for a one-dimensional matrix is undefined. The multidimensional vector matrix determinant for a twodimensional square matrix is calculated using the traditional methods. The multidimensional vector matrix determinant of a two-dimensional non-square matrix is undefined.

Hence, at first, a four-dimensional vector matrix which can be calculated determinant should be a fourdimensional vector square matrix. Secondly, commutative matrices must be square matrices with the same orders.

For instants, a four-dimensional vector square matrix $\underset{A_{m \times n \times m \times n}}{\rightarrow}=\left(a_{i 1 i 2} \overrightarrow{j_{1} j_{2}}\right) \underset{m \times n \times m \times n}{\rightarrow} \rightarrow \underset{1 \leq i_{1} \leq m}{\rightarrow}$, $1 \leq i 2 \leq n, 1 \leq j_{1} \leq m$ and $1 \leq j_{2} \leq n$.

For a four-dimensional square vector matrix $\underset{A_{m \times n \times m \times n}}{\rightarrow}$, all the elements of four vector directions where the

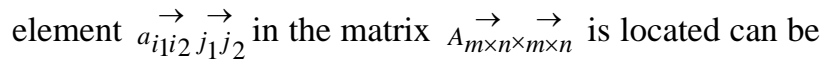
cancelled. The other elements are regularly collected in a matrix with the orders of $(m \times n-1)$ and then its determinant can be calculated. The matrix determinant can be called the cofactor of the element $\underset{a_{i 1 i 2} \vec{j}_{1} j_{2}}{\rightarrow}$, denoted as $\underset{M_{i 1 i 2}}{\overrightarrow{j_{1} j_{2}}}$, then

$$
\overrightarrow{A_{i 1 i 2}} \overrightarrow{j_{1} j_{2}}=(-1)\left[\left(i_{1}-1\right) n+i_{2}\right]+\left[\left(j_{1}-1\right) n+j_{2}\right] \underset{M_{i 1 i 2}}{\overrightarrow{j_{1} j_{2}}} \overrightarrow{{ }_{2}}
$$

$A_{i_{1} i_{2} j_{1} j_{2}}$ can be said the vector cofactor of the element $\overrightarrow{a_{i 1 i 2}} \overrightarrow{j_{1} j_{2}}$.

For example, a four-dimensional square vector matrix $\underset{2 \times 2 \times 2 \times 2}{\rightarrow}$ with the orders of two.

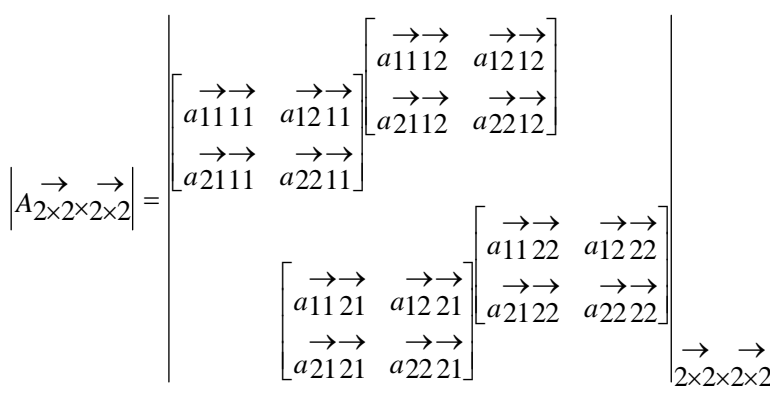

The vector cofactor of the element $\overrightarrow{a_{11} 11}$ :

$$
\overrightarrow{A_{11} 11}=(-1)[(1-1) \times 2+1]+[(1-1) \times 2+1]_{M} \overrightarrow{1111}=M \overrightarrow{1111}
$$

The vector cofactor of the element $\overrightarrow{a_{12} 22}$ :

$$
\overrightarrow{A 2122}=(-1)[(2-1) \times 2+1]+[(2-1) \times 2+2] \underset{M}{\overrightarrow{2122}} \vec{\rightarrow}=-M \overrightarrow{2122}
$$

\section{A. The Definition of Four-dimensional Vector Square Matrix Determinant}

For a four-dimensional vector square matrix, each element of any vector direction is multiplied by its vector cofactor and then all the products are added. The product can be called the four-dimensional vector square matrix determinant.

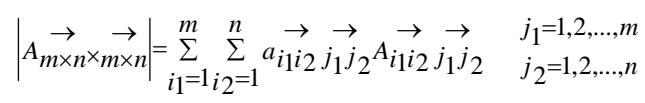

So we can prove the definition of the above formula. Due to the addition of multi-dimensional vector matrices, the four-dimensional vector square matrix determinant can be rewritten. 


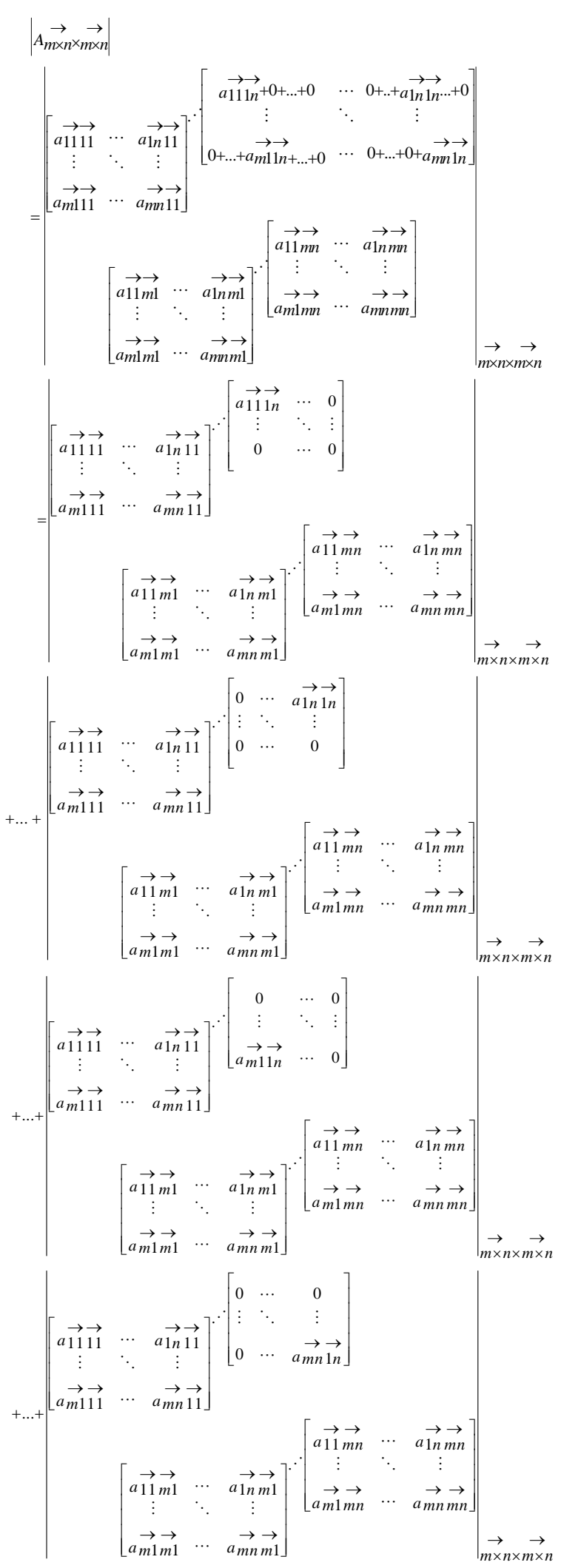

Because of the definition of four-dimensional vector square matrix determinant, we can conclude

$$
\begin{aligned}
& \left|\underset{A_{m \times n \times m \times n}}{\rightarrow} \underset{ }{\rightarrow}\right| \\
& =a_{11} \overrightarrow{1 n} A_{11} \overrightarrow{1 n}+\ldots+\overrightarrow{a_{1 n}} \overrightarrow{1 n} A_{1 n} \overrightarrow{1 n}+\ldots+\overrightarrow{a_{m 1} 1 n} \overrightarrow{A_{m 1} 1 n}+\ldots+a_{m n} \overrightarrow{n 1} A \overrightarrow{A_{m}} \overrightarrow{n 1}
\end{aligned}
$$

The four-dimensional vector matrix determinant can also be defined,

$$
|A \underset{m \times n \times m \times n}{\rightarrow}|=\sum_{j_{1}=1}^{m} \sum_{j_{2}=1}^{n} \overrightarrow{a_{i 1 i 2}} \overrightarrow{j_{1} j_{2}} \overrightarrow{A_{i 1 i 2}} \overrightarrow{j_{1} j_{2}} \quad \begin{aligned}
& i_{1}=1,2, \ldots, m \\
& i_{2}=1,2, \ldots, n
\end{aligned}
$$

If the order of $n=m$,

$$
\left|A_{m \times m \times m \times m} \rightarrow\right| \sum_{i_{1}=1}^{m} \sum_{i_{2}=1}^{m} \overrightarrow{a_{i 1} i_{2} j_{1} j_{2}} \rightarrow \overrightarrow{A_{i 1} i_{2}} \rightarrow \overrightarrow{j_{1} j_{2}} \quad \begin{aligned}
& j_{1}=1,2, \ldots, m \\
& j_{2}=1,2, \ldots, m
\end{aligned}
$$

Likewise,

$$
\left|A_{m \times m \times m \times m} \rightarrow\right| \sum_{j_{1}=1}^{m} \sum_{j_{2}=1}^{m} \overrightarrow{a_{i 1} i i_{1} j_{1} j_{2}} \rightarrow \overrightarrow{A_{i 1} i_{2}} \overrightarrow{j_{1} j_{2}} \quad \begin{aligned}
& i_{1}=1,2, \ldots, m \\
& i_{2}=1,2, \ldots, m
\end{aligned}
$$

Similarly, all elements of any vector direction in the four-dimensional vector square matrix are multiplied by the vector cofactor of corresponding elements in another vector direction and then all the products are added. The result is zero.

$$
\begin{aligned}
& \underset{a_{i 1 i 2}}{\overrightarrow{11}} A_{j_{1} j_{2}} \overrightarrow{11}+\ldots+\overrightarrow{a_{i 1 i 2}} \overrightarrow{1 n} A_{j_{1} j_{2}} \overrightarrow{1 n}+\ldots+\overrightarrow{a_{i 1 i 2} m n} \overrightarrow{A_{j_{1} j_{2}}} \overrightarrow{m n}=0 \\
& \overrightarrow{a 11} \vec{i}_{1 i 2} \overrightarrow{A_{11}} \overrightarrow{j_{1} j_{2}}+\ldots+\overrightarrow{a_{1 n} i_{i_{1} i_{2}}} \overrightarrow{A_{1 n}} \overrightarrow{j_{1} j_{2}}+\ldots+\overrightarrow{a_{m n}} \overrightarrow{i_{1} i_{2}} \overrightarrow{A_{m n}} \overrightarrow{j_{1} j_{2}}=0
\end{aligned}
$$

First, we should calculate the $|\underset{m \times n \times m \times n}{\rightarrow}|$ by the certain vector cofactor,

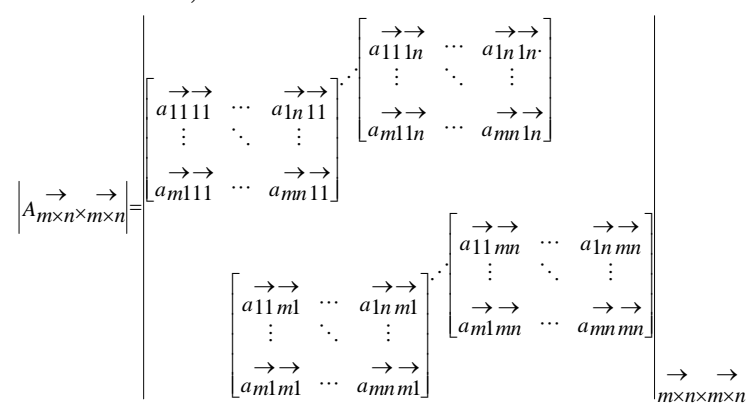

$=\overrightarrow{a_{11} 1 n} \overrightarrow{A_{11} 1 n}+\ldots+\overrightarrow{a_{1 n} 1 n} \overrightarrow{A_{1 n} 1 n}+\ldots \overrightarrow{a_{m 11 n}} \overrightarrow{A_{m 1} 1 n}+\ldots+\overrightarrow{a_{m n} 1 n} \overrightarrow{A_{m n} 1 n}$

Secondly, we replace the elements of the other certain vector direction in the sides of the above equation. That is $\overrightarrow{a_{i 12} 1 n^{n}}=a_{i 1 i 2} \overrightarrow{m 1}$.

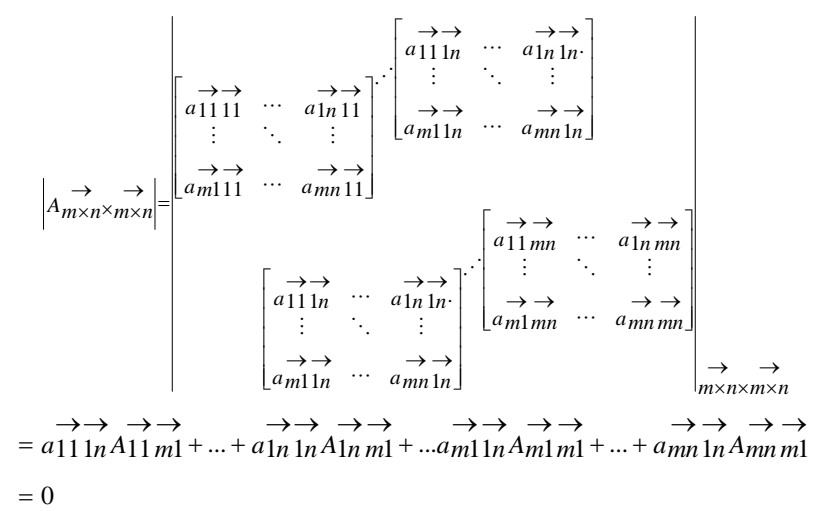

If the order of $n=m$,

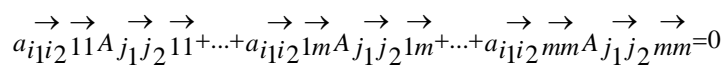

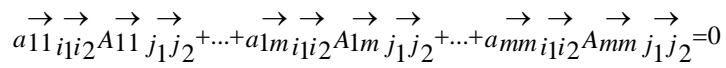


In conclusion,

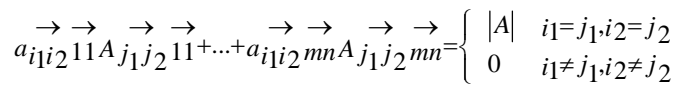

$$
\begin{aligned}
& \overrightarrow{a 11} \overrightarrow{i_{1} i_{2}} \overrightarrow{A_{11}} \overrightarrow{j_{1} j_{2}}+\ldots+a \overrightarrow{m_{i} i_{12}} \rightarrow \overrightarrow{m_{m}} \overrightarrow{j_{1} j_{2}}=\left\{\begin{array}{cc}
|A| & i_{1}=j_{1}, i_{2}=j_{2} \\
0 & i_{1} \neq j_{1}, i_{2} \neq j_{2}
\end{array}\right.
\end{aligned}
$$

\section{B. The Definition of Four-dimensional Vector Square Matrix Inverse}

The multidimensional vector matrix inverse for a onedimensional matrix is undefined. The multidimensional vector matrix inverse of a two-dimensional matrix exists if it is a square matrix and has a nonzero determinant, and is calculated using the standard means in traditional matrix math. For the four-dimensional vector matrix, each four-dimensional vector square matrix with a nonzero determinant is necessary. Firstly, we define the four-dimensional vector adjoin matrix.

The definition of four-dimensional vector adjoin matrix,

$$
\underset{A_{m \times n \times m \times n}}{\rightarrow}=\left(\overrightarrow{A_{i 1} i 2} \overrightarrow{j_{1} j_{2}}\right) \underset{m \times n \times m \times n}{\rightarrow} \underset{T}{\rightarrow}
$$

If a four-dimensional vector square matrix $\underset{A_{m \times n \times m \times n}}{\rightarrow}$ is invertible, and $\mid \underset{A_{m \times n \times m \times n}}{\rightarrow} \underset{\neq 0}{\rightarrow}$, then

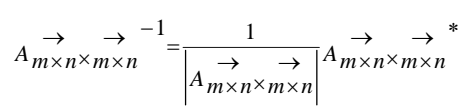

If four-dimensional vector square matrix $\underset{A_{m \times n \times m \times n}}{\rightarrow}$ is invertible, the four-dimensional vector square matrix inverse $\underset{A_{m \times n \times m \times n}}{\rightarrow} \rightarrow^{-1}$ can be existed. That is $\underset{A_{m \times n \times m \times n}}{\rightarrow} \underset{m \times n \times m \times n}{\rightarrow} \rightarrow-1$. We also calculate the determinant of the equation.

That is $|\underset{m \times n \times m \times n}{\rightarrow}|\left|A_{m \times n \times m \times n}^{\rightarrow} \underset{\sim}{\rightarrow}\right|=1(|\underset{m \times n \times m \times n}{\rightarrow \underset{ }{\rightarrow} \rightarrow}| \neq 0)$.

Contrarily, if the four-dimensional vector square matrix $|\underset{m \times n \times m \times n}{\rightarrow \underset{ }{\rightarrow}}| \neq 0$, we can find,

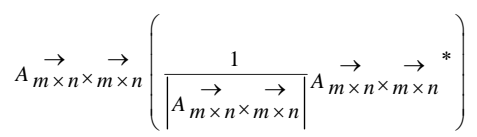

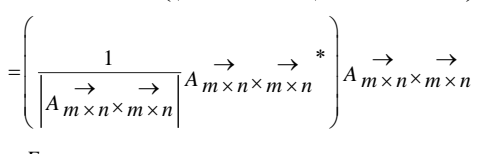

So we can also prove the formula. If a fourdimensional vector square matrix $\underset{A_{m \times n \times m \times n}}{\rightarrow}$ is invertible, and $\mid \underset{m \times n \times m \times n}{\rightarrow} \rightarrow \neq 0$, then

$$
\underset{A_{m \times n \times m \times n}}{\rightarrow}=\frac{1}{\mid \underset{m \times n \times m \times n}{\overrightarrow{A_{m}} \rightarrow}} A^{A} \underset{m \times n \times m \times n}{\rightarrow} \rightarrow^{*}
$$

If the order of $n=m$,

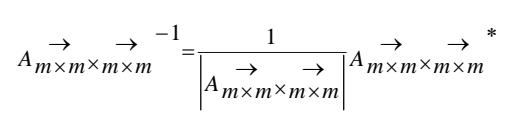

\section{THE PROPERTIES OF FOUR-DIMENSIONAL VECTOR MATRIX DETERMINANT AND INVERSE}

In the Section 3, we have defined the formula of four-dimensional vector matrix determinant and inverse. So we can conclude the properties of four-dimensional vector matrix determinant and inverse.

1. A four-dimensional vector square matrix $\underset{A_{m \times n \times m \times n}}{\rightarrow}$,

$$
\left|\underset{A_{m \times n \times m \times n}}{\rightarrow}\right|=\left|\underset{A_{m \times n \times m \times n} \rightarrow \underset{T}{\rightarrow}}{\rightarrow}\right|
$$

For a four-dimensional vector square matrix $\underset{A_{m \times n \times m \times n}}{\rightarrow}$,

$$
\begin{aligned}
& \left|A_{m \times n \times m \times n} \rightarrow\right|=\sum_{i 1}^{m} \sum_{i 2}^{n} a_{i i_{i 2}} \overrightarrow{j_{1} j_{2}} \underset{A_{i 1} i 2}{\overrightarrow{j_{1} j_{2}}} \\
& =a_{11} \overrightarrow{11} A_{11111}+\ldots+a_{1 n} \overrightarrow{11} \overrightarrow{A_{1 n}} \overrightarrow{11}+\ldots+a_{m n} \overrightarrow{11} A_{m n} \overrightarrow{11} \\
& =\sum_{j_{1}=1}^{m} \sum_{j_{2}=1}^{n} \overrightarrow{a_{i 1 i 2}} \overrightarrow{j_{1} j_{2}} \overrightarrow{A_{i 11 i}} \overrightarrow{j_{1} j_{2}} \\
& =\overrightarrow{a 11} \overrightarrow{11} A_{1111} \overrightarrow{+}+\ldots+\overrightarrow{a 111 n} A_{111 n}+\ldots+a \overrightarrow{a_{11} m n} \overrightarrow{A_{11} m n}
\end{aligned}
$$

$$
\begin{aligned}
& \left|\underset{A_{m \times n \times m \times n}}{\rightarrow}\right|^{T} \mid=\sum_{j_{1}=1}^{m} \sum_{j=1}^{n} a_{j_{1} j_{2} i i_{i 2}} \rightarrow \overrightarrow{j_{1} j_{2} i_{11 i 2}}
\end{aligned}
$$

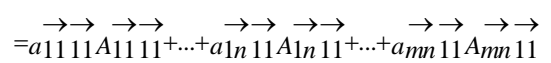

$$
\begin{aligned}
& =\sum_{i_{1}=1}^{m} \sum_{i_{2}=1}^{n} a_{j_{1} j_{2} i_{1} i_{2}} A_{j_{1} j_{2} i_{11 i 2}} \overrightarrow{ } \\
& =\overrightarrow{a_{1111}} A_{1111} \overrightarrow{a_{11}}+\ldots+\overrightarrow{a_{11}} A_{111 n}+\ldots+\overrightarrow{a_{11} m_{n}} A_{11} \overrightarrow{m n} \\
& \text { So }|\underset{m \times n \times m \times n}{\rightarrow} \rightarrow|=\mid \underset{m \times n \times m \times n}{\rightarrow} \rightarrow \text {. }
\end{aligned}
$$

2. If four-dimensional vector square matrix $\underset{A_{m \times n \times m \times n}}{\rightarrow}$ is invertible, $\lambda \neq 0$, and $\lambda \underset{m \times n \times m \times n}{\rightarrow}$ is also invertible, then

$$
\left(\lambda \underset{A_{m \times n \times m \times n}}{\rightarrow} \rightarrow{ }^{-1}=\frac{1}{\lambda} \underset{m \times n \times m \times n}{\rightarrow} \underset{\rightarrow}{\rightarrow-1}\right.
$$

We have known,

$$
\begin{aligned}
& \underset{A_{m \times n \times m \times n}}{\rightarrow} \underset{A_{m \times n \times m \times n}}{\rightarrow} \stackrel{-1}{=}_{A_{m \times n \times m \times n}}^{\rightarrow} \rightarrow^{-1} \underset{m \times n \times m \times n}{\rightarrow}=E
\end{aligned}
$$

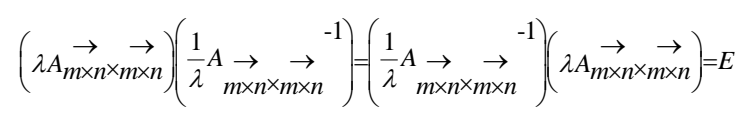

If $\lambda \underset{m \times n \times m \times n}{\rightarrow}$ is invertible,

$$
\left(\lambda A_{m \times n \times m \times n}^{\rightarrow} \rightarrow{ }^{-1}=\frac{1}{\lambda} A \underset{m \times n \times m \times n}{\rightarrow} \underset{-1}{\rightarrow} .\right.
$$


3. If four-dimensional vector square matrix $\underset{A_{m \times n \times m \times n}}{\rightarrow}$ and $\underset{A_{m \times n \times m \times n}}{\rightarrow} \rightarrow^{T}$ are both invertible, then

$$
\left(\underset{A_{m \times n \times m \times n}}{\rightarrow}\right)^{T}=\left(\underset{A_{m \times n \times m \times n}}{\rightarrow}\right)^{-1}
$$

If $\underset{A_{m \times n \times m \times n}}{\rightarrow}$ is invertible,

$$
\underset{A_{m \times n} \times m \times n}{\rightarrow} \underset{m \times n \times m \times n}{\rightarrow} \underset{A_{m \times n \times m \times n}^{-1}}{\rightarrow} \underset{A_{m \times n \times m \times n}}{\rightarrow} \underset{-1}{\rightarrow}=E
$$

We calculate the transpose of the equation,

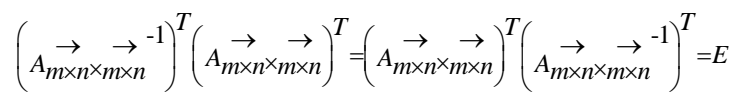

If the four-dimensional vector matrix $\left(\underset{A_{m \times n \times m \times n}}{\rightarrow}\right)^{T}$ is invertible,

$$
\left(\underset{A_{m \times n \times m \times n}}{\rightarrow}\right)^{T}=\left(\underset{A_{m \times n \times m \times n}}{\rightarrow}\right)^{-1}
$$

4. If four-dimensional vector matrix $\underset{A_{m \times n \times m \times n}}{\rightarrow}$ and $\underset{B_{m \times n \times m \times n}}{\rightarrow}$ are both invertible and four-dimensional vector square matrix $\underset{A_{m \times n \times m \times n}}{\rightarrow} \underset{m \times n \times m \times n}{\rightarrow}$ is also invertible, then

$$
\left(\underset{A_{m \times n \times m \times n}}{\rightarrow} \underset{m \times n \times m \times n}{\rightarrow}\right)^{-1}=\underset{B \rightarrow m \times n \times m \times n}{\rightarrow} \underset{A_{m \times n \times m \times n}}{\rightarrow} \underset{-1}{\rightarrow}
$$

If four-dimensional vector square matrix $\underset{A_{m \times n \times m \times n}}{\rightarrow} \underset{m}{\rightarrow}$ and $\underset{B_{m \times n}}{\rightarrow} \underset{m \times n}{\rightarrow}$ are both invertible,

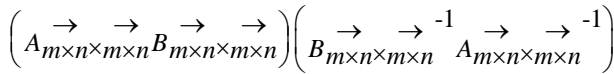

$$
\begin{aligned}
& =\underset{A_{m \times n \times m \times n}}{\rightarrow}\left(\underset{B_{m \times n \times m \times n}}{\rightarrow} \underset{m \times n \times m \times n}{\rightarrow} \underset{-1}{\rightarrow} \underset{A_{m \times n \times m \times n}}{\rightarrow} \underset{-1}{\rightarrow}\right. \\
& =\underset{m \times n \times m \times n}{\rightarrow} \underset{m \times n \times m \times n}{\rightarrow} \underset{m \times n \times m \times n}{\rightarrow} \rightarrow^{-1} \\
& =\underset{m \times n \times m \times n}{\rightarrow} \underset{A_{m \times n \times m \times n}}{\rightarrow} \underset{m}{\rightarrow-1} \\
& =E
\end{aligned}
$$

Similarly,

$$
\left(\underset{B_{m \times n \times m \times n}}{\rightarrow} \rightarrow_{m \times n \times m \times n}^{-1} A \underset{-1}{\rightarrow}(\underset{m \times n \times m \times n}{\rightarrow} \underset{m \times n \times m \times n}{\rightarrow} \rightarrow \underset{ }{\rightarrow})=E\right.
$$

If the four-dimensional vector matrix $\underset{A_{m \times n} \times m \times n}{\rightarrow} \overrightarrow{m \times n \times m \times n} \underset{m}{\rightarrow}$ is invertible,

$$
(\underset{m \times n \times m \times n}{\rightarrow} \overrightarrow{m \times n \times m \times n})^{-1}=\underset{B_{m \times n} \times m \times n}{\rightarrow} \underset{A_{m \times n \times m \times n}}{\rightarrow} \underset{-1}{\rightarrow}
$$

5. If four-dimensional vector square matrix $\underset{A_{m \times n \times m \times n}}{\rightarrow} \underset{\text { is }}{\rightarrow}$ invertible, $\left(\underset{A_{m \times n \times m \times n}}{\rightarrow}-1\right) \underset{m \times n \times m \times n}{\rightarrow}=U N I T$.

In traditional matrix mathematics, if a matrix possesses an inverse and that matrix is multiplied by its inverse, the product is an identity matrix with the same dimensions.

Because multidimensional vector matrices are a concatenation of two-dimensional matrices, if a fourdimensional vector matrix has an inverse and that fourdimensional vector matrix is multiplied by its inverse, then the product will be a four-dimensional vector identity matrix with the same dimensions.

Due to the definition of four-dimensional vector matrix inverse $(4)$ and $|\underset{m \times n \times m \times n}{\rightarrow}| \neq 0$, if this matrix $\underset{A_{m \times n \times m \times n}}{\rightarrow} \rightarrow *$ is the four-dimensional vector adjoin matrix, we can conclude

$$
\begin{aligned}
& \left(\underset{A_{m \times n \times m \times n}}{\rightarrow} \rightarrow^{-1}\right) \underset{m \times n \times m \times n}{\rightarrow}
\end{aligned}
$$

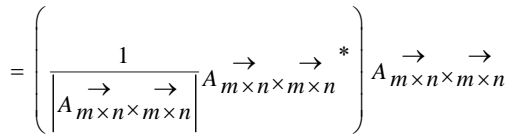
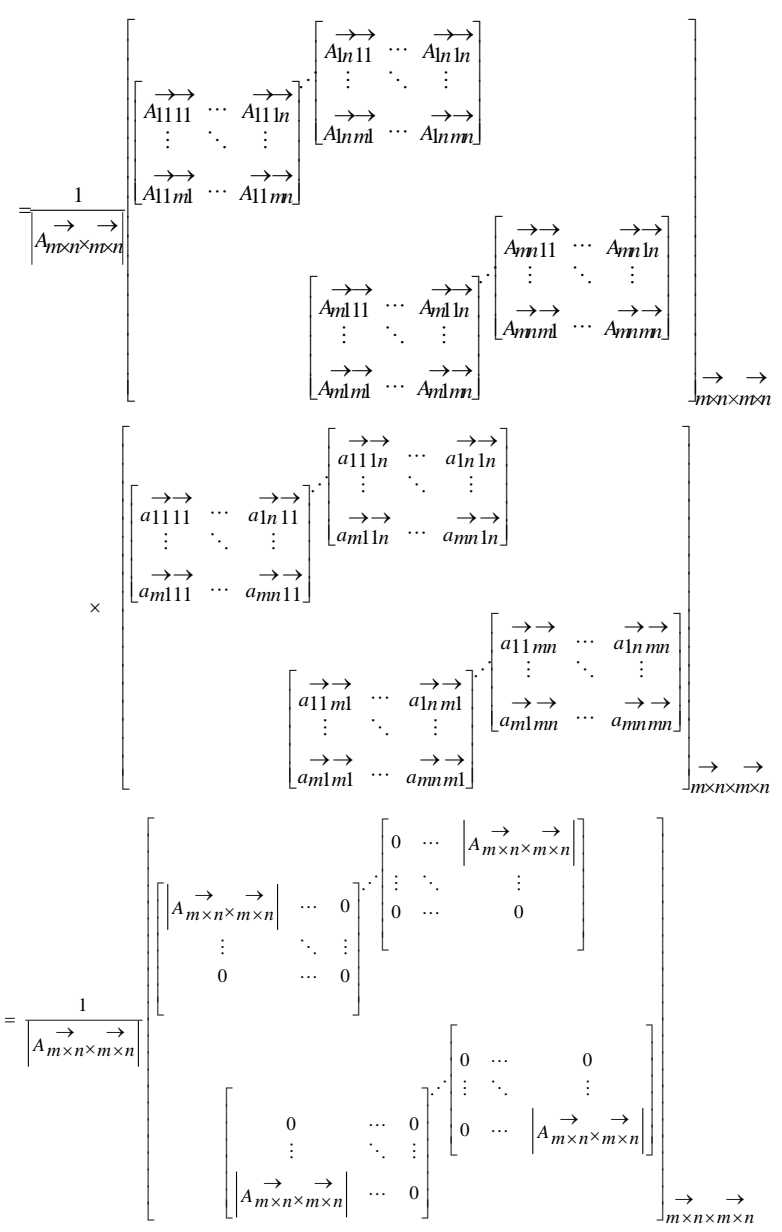

$=U N I T$

Due to the multiplication of multi-dimensional vector matrices (1) and the formula of four-dimensional vector 
matrix determinant (2) and (3), that is $\left.\left(\underset{A_{m \times n \times m \times n}}{\rightarrow}\right)^{-1}\right) \underset{m \times n \times m \times n}{\rightarrow}=U N I T$.

For example, the four-dimensional vector matrix $\underset{2 \times 2 \times 2 \times 2}{\rightarrow}$ with two orders is given. By means of the program's operation, we can calculate the fourdimensional vector inverse matrix.

$$
\begin{aligned}
& \underset{A_{2 \times 2 \times 2 \times 2}}{\rightarrow} \underset{2 \times 2 \times 2 \times 2}{\rightarrow} \rightarrow^{-1}
\end{aligned}
$$

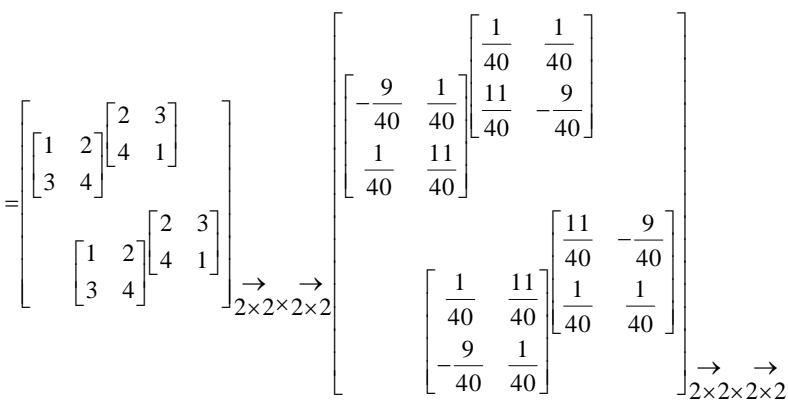

$$
\begin{aligned}
& =\left[\begin{array}{rl}
{\left[\begin{array}{ll}
1 & 0 \\
0 & 0
\end{array}\right]\left[\begin{array}{ll}
0 & 1 \\
0 & 0
\end{array}\right]} & {\left[\begin{array}{ll}
0 & 0 \\
1 & 0
\end{array}\right]\left[\begin{array}{ll}
0 & 0 \\
0 & 1
\end{array}\right]}
\end{array}\right] \underset{2 \times 2 \times 2 \times 2}{\rightarrow \underset{2}{\rightarrow}} \\
& =U N I T
\end{aligned}
$$

6. The determinant of four-dimensional vector identity matrix $\mid \underset{A_{m \times n \times m \times n}}{\rightarrow} \rightarrow$ is 1 .

In traditional matrix mathematics, if a matrix is an identity matrix, the determinant of two-dimensional matrix is 1 .

Similarly, multidimensional vector matrices are a concatenation of two-dimensional matrices, if a fourdimensional vector matrix is a four-dimensional vector identity matrix, the result of four-dimensional vector identity matrix determinant is 1 . That is $|\underset{m \times n \times m \times n}{\vec{A}}|=1$.

For example,

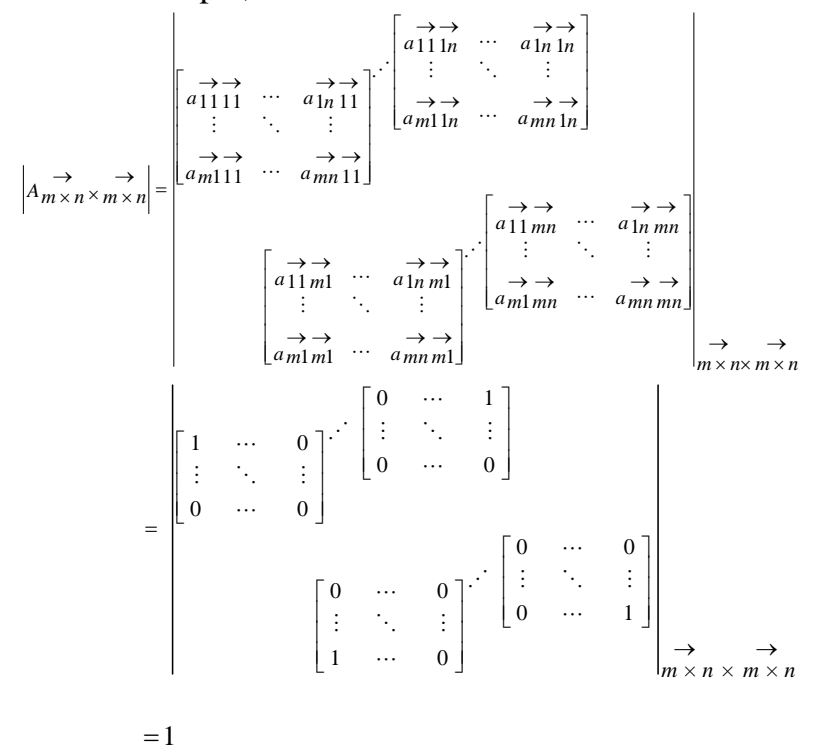

7. The other properties of multidimensional vector matrix determinant and inverse.

There are still many properties of two-dimensional matrix that can be extend to the four-dimensional vector matrix.

If all the elements of any vector direction are zero in a four-dimensional vector square matrix $\underset{A_{m \times n \times m \times n}}{\rightarrow}$, then $\left|\underset{A_{m \times n \times m \times n}}{\rightarrow}\right|=0$.

If one vector direction is proportional to another vector direction of a four-dimensional vector square matrix $\underset{A_{m \times n \times m \times n}}{\rightarrow}$, then $\mid \underset{A_{m \times n \times m \times n}}{\rightarrow} \rightarrow=0$.

If one vector direction is a linear combination of one or more other vector directions of a four-dimensional vector square matrix $\underset{A_{m \times n \times m \times n}}{\rightarrow}$, then $\mid \underset{A_{m \times n \times m \times n}}{\rightarrow} \rightarrow=0$.

If two vector directions of a four-dimensional vector square matrix $\underset{A_{m \times n \times m \times n}}{\rightarrow}$ are interchanged, the sign of the

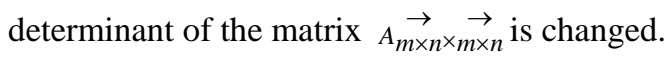

A four-dimensional vector square matrix $\underset{A_{m \times n \times m \times n}}{\rightarrow} \rightarrow$ inverse which it is an invertible matrix can be unique.

If four-dimensional vector square matrix $\underset{A_{m \times n \times m \times n}}{\rightarrow}$ is invertible, $\left(\underset{A_{m \times n \times m \times n} \rightarrow \underset{m}{\rightarrow}}{\rightarrow-1}=\underset{m \times n \times m \times n}{\rightarrow}\right.$.

There are various properties of four-dimensional vector matrix determinant and inverse to prove the correctness of four-dimensional vector matrix determinant and inverse definition in this paper. Meanwhile, we run successfully the corresponding program to verify the definition of the four-dimensional vector matrix determinant and inverse.

\section{CONCLUSION}

On the basis of newly operation laws of multidimensional vector matrix, we define the fourdimensional vector matrix determinant, inverse and related properties in this model. We also prove the correctness of these associated formulas by mathematics and program. In this program, followed by the definitions and certification of four-dimensional vector matrix, we have successfully run related program and get the rational result which the four-dimensional vector matrix $\underset{A_{m \times n \times m \times n}}{\rightarrow}$ multiplied by the four-dimensional vector matrix inverse $\underset{A_{m \times n \times m \times n}}{\rightarrow}$ is equal to the fourdimensional vector identity matrix.

In this paper, we have introduced mainly the theory of multi-dimensional vector matrix, the four-dimensional vector matrix determinant and inverse. The future work is to extend the four-dimensional vector matrix inverse $\underset{A_{m \times n} \times m \times n}{\rightarrow} \underset{-1}{\rightarrow}$ to multidimensional vector matrix inverse $\underset{A_{m_{1}} \times m_{2} \ldots \times m_{n} \times m_{1} \times m_{2} \ldots m_{n}}{\rightarrow} \stackrel{-1}{\rightarrow}$. We will apply adopted theories and definitions on multidimensional vector 
$\operatorname{matrix} \underset{A_{m_{1} \times m_{2} \ldots . .} \times m_{n \times m_{1} \times m_{2} \ldots . . .}}{\rightarrow}$

\section{ACKNOWLEDGMENT}

This work is sponsored by the National Science Foundation of China under Grant 60911130128 and 60832002.

\section{REFERENCES}

[1] Franklin, Joel L. [2000] Matrix Theory. Mineola, N.Y.: Dover.

[2] Ashu M.G. Solos. Multidimensional matrix mathematics: multidimensional matrix transpose, symmetry, ant symmetry, determinant, and inverse, part 4 of 6 . Proceedings of the World Congress on Engineering 2010, vol.3, WEC 2010, June 30-July 2, 2010, London, U.K.

[3] Ahmed, N., Natarajan, T. and Rao, K. R. On image processing and a discrete cosine transform. IEEE Trans. Compute, 1974, 23, 90-93.

[4] A J Sang, M S Chen, H X Chen, L L Liu and T N Sun. Multi-dimensional vector matrix theory and its application in color image coding. The Imaging Science Journal vol.58, no.3, June 2010, pp.171-176(6).

[5] Liu, L.L, Chen, H.X, Sang, A.J, Sun, T.N. “4D order-4 vector matrix DCT integer transform and its application in video code,” Imaging Science Journal, the vol. 58, no. 6, December 2010, pp.321-330 (10).

[6] I. Gessel and D. Stanton, Application of q-Lagrange inversion to basic hyper geometric series, Trans. Amer. Math. Soc. 277(1983), 173-203.

[7] Christian Krattenthaler and Michael Schlosser, "A New Multidimensional Matrix Inverse with Applications to Multiple q-series”, Discrete Mathematics, Volume 204, Issues 1-3, 6 June 1999, Pages 249-279.

[8] J. Riordan, Combinatorial identities, J. Wiley, New York, 1968.

[9] H.W. Gould, “A series transformation for finding convolution identities”, Duke Math.J.28 (1961), 193-202.

[10] H.W. Gould, “A new convolution formula and some new orthogonal relations for inversion of series”, Duke Math.J.29 (1962), 393-404.

[11] H.W. Gould, "A new series transform with application to Bessel, Legrende, and Tchebychev polynomials”, Duke Math. J. 31(1964), 325-334.

[12] H.W. Gould, "Inverse series relations and other expansions involving Humbert polynomials”, Duke Math.J.32 (1965), 691-711.

[13] H.W. Gould and L.C. Hsu, "Some new inverse series relations”, Duke Math.J.40 (1973), 885-891.

[14] G.E. Andrews, “Connection coefficient problems and partitions”, D. Ray- Chaudhuri, ed., Proc. Symp. Pure Math, vol.34, Amer. Math. Soc., Providence, R. I., 1979, $1-24$.

[15] W.N. Bailey, "Some identities in combinatory analysis", Proc. London Math. Soc. (2) 49 (1947), 421-435.

[16] W.N. Bailey, "Identities of the Roger-Ramanujan type", Proc. London Math. Soc. (2) 50 (1949), 1-10.

[17] G. Gasper, "Summation, transformation and expansion formulas for bibasic series”, Trans. Amer. Soc. 312(1989), 257-278.

[18] M. Rahman, "Some quadratic and cubic summation formulas for basic hyper geometric series”, Can. J. Math. 45 (1993), 394-411.

[19] C. Krattenthaler, “A new matrix inverse”, Proc. Amer. Math. Soc. 124 (1996), 47-59.
[20] L. Carlitz, "Some inverse relations", Duke Math. J. 40(1973), 893-901.

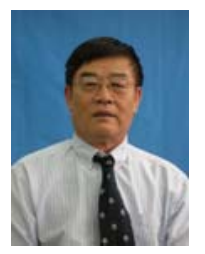

Hexin Chen is a Professor in the School of Communication Engineering in Jilin University. He received Ph.D. degree from Jilin University in 1990. From1987-1988, he worked as visiting scholar at University of Alberta, Canada. In 1993 he worked as visiting professor at University of Tampere, Finland. His research interests include multidimensional signal processing, video and image coding and decoding, video communication, multimedia database technology.

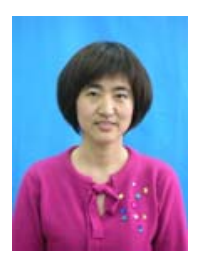

Aijun Sang is now a vice professor of the School of Communication Engineering in Jilin University. She received her Bachelor Degree at Huazhong University of Science and Technology, Master Degree at Dalian University of Technology, and Ph.D. Degree at Jilin University. Her research interests include multidimensional signal processing, video and image coding.

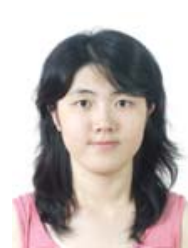

Haojing Bao is now a postgraduate of the School of Communication Engineering in Jilin University. She received her Bachelor Degree at Jilin University in 2009. Her research interests include multidimensional signal processing, video and image coding. 